\title{
Dyskinesie der Abdominalmuskulatur bei Hypochondrie
}

\author{
G. E. Feurle, K.-H. Krause und M. Grußendorf \\ Medizinische Poliklinik und Neurologische Klinik der Universität Heidelberg
}

Bei einer 73jährigen Frau mit chronischer Krankheitsfurcht, Depression und abdominellen Mißempfindungen wie Koliken, Blähungen und wechselnder Stuhlkonsistenz wurden die Diagnosen "irritables Kolon" und "Hypochondrie" gestellt. Die Patientin nahm wegen ihrer Beschwerden über 10 Jahre hinweg regelmäßig Psychopharmaka ein, vor allem Diazepine und Amitriptylin. Vor 3 Jahren begannen wellenartige, repetitiv ablaufende Bewegungen der Bauchdecke, als deren Ursache eine enterale Hyperperistaltik bei chronischem Subileus angenommen wurde. Tatsächlich ergab die Diagnostik an Dünn- und Dickdarm mittels Röntgen und Endoskopie keine Abnormität, vielmehr fanden sich wellenförmige Kontraktionen der Bauchdeckenmuskulatur, die sich im Elektromyogramm nachweisen ließen. Als Ursache war am ehesten der langjährige Psychopharmaka-Abusus anzunehmen. Bei abdominellen Symptomen ist nicht nur der MagenDarm-Trakt, sondern auch die Bauchdecke in Betracht zu ziehen.

\section{Dyskinesia of the abdominal musculature in hypo-} chondria

A 73-year-old woman with a history of chronic hypochondria, depression and abdominal symptoms, such as colics, flatulence and changing fecal consistency, was diagnosed as having an "irritable colon" syndrome and "hypochondria «. Over a period of ten years she had been taking regularly various psychoactive drugs, especially diazepins and amitryptiline. Three years ago she began to have wave-like movements of the abdominal wall, interpreted as intestinal hyperperistalsis with chronic subileus. Radiological and endoscopic examinations were normal, but wavelike contractions of the abdominal musculature were demonstrated on electromyography. The most likely cause is the chronic abuse of psychoactive drugs over many years. In case of abdominal symptoms attention should be paid to the abdominal wall, not only the gastrointestinal tract.
Bei anhaltender wellenförmiger Bewegung der Bauchoberfläche denkt man zunächst an Hyperperistaltik beim chronischen Subileus. Im folgenden berichten wir über eine alternative Ursache.

\section{Kasuistik}

Eine 73jährige Frau wurde wegen unklarer abdomineller Symptomatik überwiesen. Zur Vorgeschichte gab sie an, seit etwa 10 Jahren an Blähungen, kolikartigen Bauchschmerzen und Wechsel von Verstopfung und Durchfall zu leiden. Sie sei praktisch nie beschwerdefrei. Mehrfache endoskopische und röntgenologische Untersuchungen des Magen-Darm-Traktes hätten nie einen pathologischen Befund erbracht. Außerdem klagte die Patientin darüber, daß sie jeden Lebensmut verloren habe, und äußerte seit Jahren bei jeder Gelegenheit Angst vor einer bösartigen Erkrankung im Bauch. Aufgrund dieser Symptome war von den Ärzten, die die Patientin aufgesucht hatte, die Diagnose "Syndrom des irritablen Kolons bei depressiv-hypochondrischem Zustand " gestellt worden. Infolge dieser Stimmungslage nahm die Patientin seit etwa 10 Jahren regelmäßig Psychopharmaka ein, im wesentlichen Chlordiazepoxid, Amitriptylin, Diazepam, Bromazepam, Temazepam, Dikaliumclorazepat, Flunitrazepam. Im Jahre 1982 wurde einige Male wöchentlich 1,5 mg des Neuroleptikums Fluspirilen injiziert und im Jahre 1984 kurzfristig 2 mg Haloperidol mit Steigerung der Dosis auf $8 \mathrm{mg}$. In der Zeitspanne vom 1. 6. 1984 bis zum 31. 3.

Dtsch. med. Wschr. 112 (1987), 139-141

(c) 1987 Georg Thieme Verlag Stuttgart · New York
1985 ließen sich die rezeptierten Psychopharmaka durch Rücksprache bei der Krankenkasse quantitativ erfassen. Durch Multiplikation der Angaben in Tabelle 1 mit dem Faktor 12 läßt sich ein Anhaltspunkt für die Menge der insgesamt eingenommenen Psychopharmaka gewinnen.

Im Juli 1983 begannen unaufhörliche, wellenförmig ablaufende Bauchbewegungen, die später mit ziehenden Schmerzen verbunden waren. Diese Bewegungen verstärkten sich in den letzten Monaten, auswärts wurde dieses Phänomen als eine durch die Bauchwand sichtbare Hyperperistaltik interpretiert.

Bei der Untersuchung wog die Patientin $48 \mathrm{~kg}$ bei $159 \mathrm{~cm}$. Sie klagte über wellenförmige Bewegungen im Bauch mit Schmerzen sowie über Blähungen, Appetitverlust und Schlafstörungen. Bewe-

Tab. 1, Kumulative Dosis einiger (möglicherweise nicht aller) während 10 Monaten rezeptierter Psychopharmaka

\begin{tabular}{|ll|}
\hline Phenobarbital & $0,45 \mathrm{~g}$ \\
Bromazepam & $0,12 \mathrm{~g}$ \\
Temazepam & $1,2 \mathrm{~g}$ \\
Tilidin & $0,5 \mathrm{~g}$ \\
Diazepam & $0,4 \mathrm{~g}$ \\
Piracetam & $1,488 \mathrm{~g}$ \\
Amitriptylin & $1,25 \mathrm{~g}$ \\
Flunitrazepam & $0,16 \mathrm{~g}$ \\
Levomepromazin & $0,4 \mathrm{~g}$ \\
Maprotilin & $6,25 \mathrm{~g}$ \\
\hline
\end{tabular}


gungsstörungen anderer Art, zum Beispiel eine orolinguofaciale Hyperkinesie oder choreoathetotische Bewegungen, waren nicht zu beobachten. Die Atmung war regelmäßig. Bei Inspektion des Abdomens beobachtete man eine unregelmäßige, wellenförmig ablaufende Bewegung, die nur bei längerer Beobachtung eine gewisse Stereotypie erkennen ließ. Sie begann ruckartig unterhalb des Rippenbogens im rechten Oberbauch mit einer Frequenz von etwa $30-50 / \mathrm{min}$ und pflanzte sich dann wellenförmig über das gesamte Abdomen hin fort. Das Abdomen war nie in Ruhe, die Bewegung verstärkte sich beim Zurückschlagen der Bettdecke, hörte aber auch dann nicht auf, wenn die Patientin abgelenkt wurde. Kurzfristig sistierte die Bewegung, wenn beispielsweise die Bauchmuskeln beim Heben der Beine angespannt wurden. Bei Seiten- oder Rükkenlage war festzustellen, daß auch die Muskeln der Regio lumbalis an der Bewegung teilhatten. Bei Palpation waren die Bewegungen ebenfalls festzustellen. Der Bauch war weich, nicht vorgewölbt, die Darmgeräusche waren normal, ein Druckschmerz oder eine Resistenz nicht feststellbar. Der übrige körperliche Befund war völlig unauffällig.

Die neurologische Untersuchung ergab bis auf die rhythmischen Kontraktionen der Bauchmuskulatur einen Normalbefund. Die Bauchdeckenmuskulatur war nicht spastisch, die Bauchhautreflexe waren wegen der ständigen Bewegungsunruhe nicht sicher beurteilbar. Das EEG war unauffällig. Während einer Beobachtungszeit von 10 Monaten war eine geringe Zunahme der Dyskinesie zu

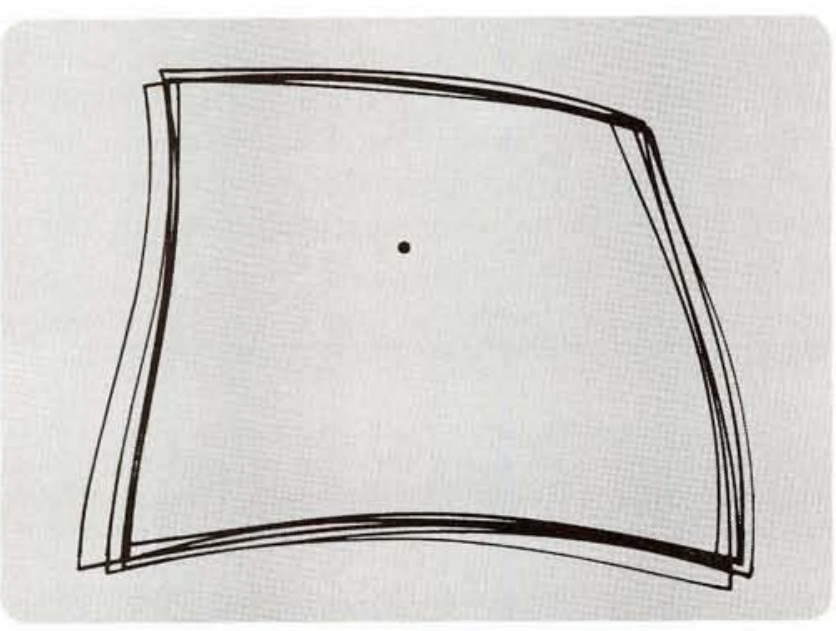

Abb. 1. Bewegungen der Außenkontur eines mit schwarzem Pflaster auf den Bauch der Patientin geklebten Vierecks, gezeichnet nach Vergrößerungen von sechs aufeinanderfolgenden Bildabschnitten eines Super-8-Films. Die Markierung innerhalb des Vierecks gibt die Position des Nabels wieder. beobachten. Therapieversuche mit Tiaprid, Clonazepam und Haloperidol blieben erfolglos.

Eine Dokumentation der Dyskinesie der Abdominalwand erfolgte mittels Super-8-Kamera, nachdem auf den Bauch der Patientin ein Viereck mit schwarzem Pflaster geklebt worden war. Bei Vergrößerung und Aufzeichnung von sechs Bildern läßt sich ein Eindruck der Bewegungen der Bauchdecke gewinnen (Abbildung 1).

Eine elektromyographische Untersuchung (Disa, System 1500) erfolgte synchron mit zwei Nadelelektroden aus dem M. transversus abdominis rechts und links. Es kamen rhythmische Entladungen von normal konfigurierten gruppierten Muskelaktionspotentialen mit wechselnden Frequenzen um 40 bis $45 / \mathrm{min}$ zur Darstellung, wobei die Erregung der rechtsseitigen Bauchmuskeln derjenigen der linksseitigen jeweils unmittelbar vorausging (Abbildung 2). Eine intravenöse Gabe von Biperiden hatte keinen Einfluß auf die Muskelkontraktionen. Eine erneute Röntgen-Dünndarmpassage verlief regelrecht.

\section{Diskussion}

Das Elektromyogramm bestätigte den klinischen Eindruck, daß es sich um eine unregelmäßig erscheinende, letztlich aber doch stereotype Kontraktion der Bauchdeckenmuskeln und somit um eine Dyskinesie handelte. Eine Hyperperistaltik der Darmschlingen war nicht nachzuweisen. Bei der Suche nach der Ursache der Dyskinesie war als erstes an die Nebenwirkungen von Pharmaka zu denken:

Anhaltende, stereotype, repetitiv aber doch arrhythmisch ablaufende Hyperkinesien besonders der Zunge, des Mundes, der Kau- und Wangenmuskulatur sind häufig beschriebene Nebenwirkungen nach langjähriger Therapie mit Neuroleptika $(5,6)$.

Diese Bewegungsstörungen werden als Spätdyskinesie bezeichnet (2). Unsere Patientin nahm Neuroleptika soweit retrospektiv eruierbar - allerdings nur während weniger Wochen ein. Im allgemeinen geht der Spätdyskinesie eine etwa dreijährige Behandlung mit Neuroleptika voraus. Irreversible Spätdyskinesien sind aber auch schon nach einigen Monaten Neuroleptikatherapie beschrieben (3); die Frage nach dem pathogenetischen Zusammenhang zwischen der Einnahme der nur kurzfristig verabreichten Neuroleptika und der Dyskine-

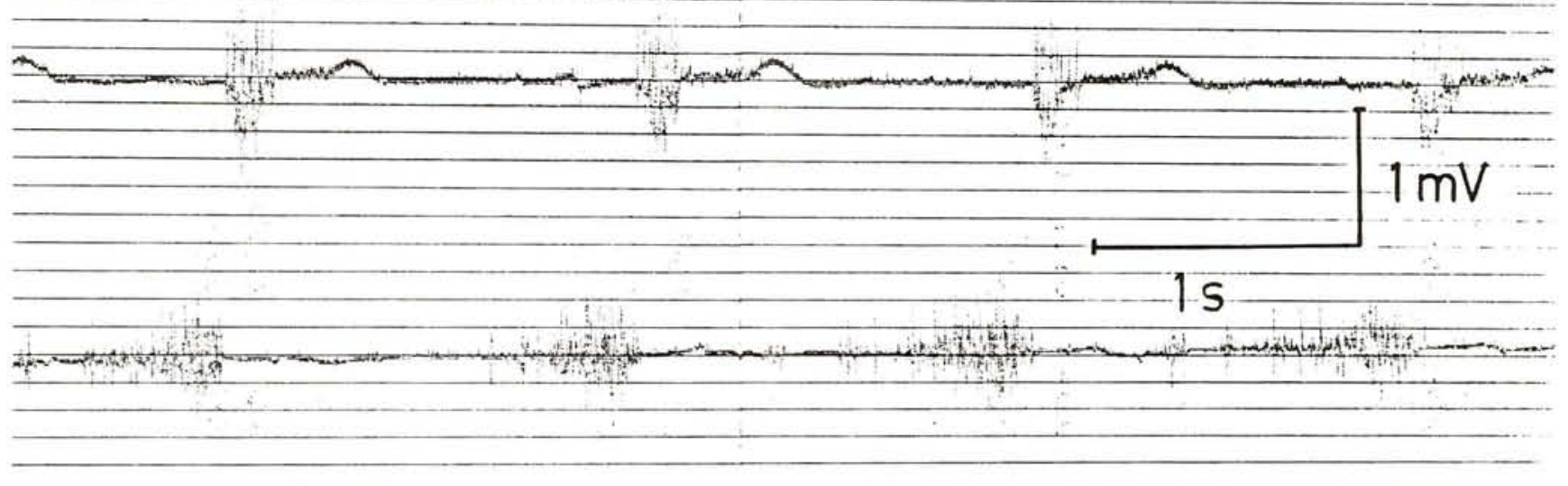

Abb. 2. Elektromyogramm aus dem M. transversus abdominis links (oberer Kanal) und rechts (unterer Kanal). 
sie der Bauchmuskulatur bei unserer Patientin muß offenbleiben.

Sehr selten sind Dyskinesien - des Mundes und des Gesichtes - auch nach Amitriptylin beobachtet worden (1). Ein Anhalt für sonstige systemische oder erbliche extrapyramidal-motorische Störungen fand sich nicht. Die Annahme einer Mitbeteiligung der quergestreiften Abdominalmuskulatur an dem vermuteten Syndrom des irritablen Kolons blieb spekulativ. Eine rein psychogene Genese hielten wir bei der komplexen und willkürlich nicht nachzuvollziehenden Bewegungsfolge ebenfalls für unwahrscheinlich. Einigermaßen plausibel ließen sich einige der Symptome dem Krankheitsbegriff der Hypochondrie zuordnen:

Die Erscheinungen spielten sich tatsächlich unterhalb der Rippenknorpel ab, an denen die Bewegungsstörung sogar ihren Ausgang nahm. Weiterhin fanden sich sowohl die Erscheinungen des Hypochondriebegriffs der Antike, wie Blähungen, Aufstoßen, Bauchweh, Furcht und Traurigkeit (Galen, Diokles von Karystos) als auch das Charakteristikum der heutigen Definition der Hypochondrie, der einer Nosophobie (Übersicht: 4). Das "Syndrom des irritablen Kolons " hat offensichtlich die Nachfolge der alten Hypochondrie angetreten.

Die Ursache der Dyskinesie der Bauchmuskulatur unserer Patientin bleibt aber auch bei dieser Deutung unklar. Am ehesten ist unseres Erachtens der langjährige Abusus von Psychopharmaka als Ursache anzunehmen. Über eine derartige, auf die Bauchmuskulatur beschränkte Dyskinesie ist unseres Wissens weder als Psychopharmakafolge noch überhaupt je berichtet wor- den. Jedenfalls blieb eine Suchanfrage mit MEDLARS-2 über die Zeitspanne 1966 bis 1985 negativ. Die Bewegungen der Bauchoberfläche hatten mehrere Ärzte zu der Diagnose "chronischer Subileus mit Hyperperistaltik " veranlaßt. Diese Fehlbeurteilung führte zu unnötigen Röntgen-, Endoskopie- und Laboratoriumsuntersuchungen. Bei abdominellen Symptomen sollte man nicht nur den Intestinalorganen, sondern auch der Bauchdecke Aufmerksamkeit widmen.

\section{Literatur}

(1) Böning, J.: Zentralmotorische und extrapyramidale Nebenwirkungen unter

Therapie mit Antidepressiva. Fortschr. Neurol. Psychiat. 50 (1982), 35.

(2) Brücher, K.: Die Spätdyskinesien Klinik, Pathophysiologie, Therapie. Dtsch. med. Wschr. 109 (1984) 1533.

(3) Evans, J. H.: Persistant oral dyskinesia in treatment with phenothiazine derivatives. Lancet 1965/1, 458 .

(4) Fischer-Homberger, E.: Hypochondrie (Huber: Bern-Stuttgart-Wien 1970). (5) Klawans, H. L., C. G. Götz, S. Perlik: Tardive dyskinesia. Review and update. Amer. J. Psychiat. 137 (1980), 900. (6) The Task Force on late Neurologica Effects of Antipsychotic Drugs: Tardive dyskinesia. Summary of the Task Force Report of the American Psychiatric Association. Amer. J. Psychiat. 137 (1980), 1163.

Prof. Dr. G. E. Feurle

I. Medizinische Abteilung

Stadtkrankenhaus

5450 Neuwied, Marktstr. 74

Privatdozent Dr. K.-H. Krause

Neurologische Universitätsklinik 6900 Heidelberg 1, Voßstr. 2

Dr. M. Grußendorf

Zentrum für Innere Medizin der Universität 4000 Düsseldorf 1, Moorenstr. 5 